\title{
Innovations
}

\section{Self-monitoring checklists: A tool for connecting training to practice}

\section{Authors}

M. Courtney Hughes ${ }^{1 *}$; Emaley B. McCulloch'; Elise G. Valdes ${ }^{\top}$

\section{Abstract}

A limitation of training is the gap between the knowledge learned in training and the behavior put into practice. Skills checklists are helpful for reducing errors and increasing adherence to safety and may be one tool useful for bridging that gap. There is little research examining the role of skills checklists with self-reflection completed independently, or "self-monitoring checklists," for increasing knowledge and improving skills in areas requiring attentional behavior in healthcare. Two randomized controlled studies incorporated self-monitoring checklists along with online training in cultural competence and integrated care, respectively, for health professionals. At least ninety percent of participants in both studies found self-monitoring checklists to be helpful. Healthcare educators and practitioners should consider self-monitoring checklists as an additional tool for online training when developing educational strategies for healthcare professionals.

\section{Keywords}

skills checklist, self-monitoring, online training, education, memory

\section{Introduction}

There is often a gap between skills training and skills practice for healthcare professionals. ${ }^{\prime}$ Sometimes educators incorrectly assume that learners will automatically generalize skills taught in a structured context. For example, an educator may assume that teaching communication skills results in his/ her student's improved communication with others. However, educators cannot trust this underlying assumption, also known as "train and hope".,2,3 Instead, educators must take measures to ensure the knowledge and skills taught in training translate into actual changes in behavior.

One strategy for helping to bridge the gap between training and practice is using skills checklists. A skills checklist is "a list of action items arranged in a systematic manner that allows the user to record the completion of the individual item"4, and skills checklists are typically completed by a health professional's supervisor. ${ }^{5.6}$ Skills checklists, used successfully for more than 70 years in
'Relias Institute, Cary, NC, United States of America

*Email: mchughes@u.washington.edu

M. Courtney Hughes ID https://orcid.org/0000-0002-8699-5701

Elise G. Valdes (iD https://orcid.org/0000-0002-0648-5657
Cite this article as:

Hughes MC, McCulloch EB, Valdes EG. Self-monitoring checklists: A tool for connecting training to practice. Innovations in Global Health Professions Education. 2018:4. https://dx.doi.org/10.20421/ighpe2018.04

This is an open access article distributed under the terms of the Creative Commons Attribution license CC BY 4.0, which permits unrestricted use, distribution and reproduction in any medium, provided the original work is properly cited. 
the aviation industry to ensure the consistent application of safety standards, can be effective for increasing compliance with healthcare tasks, with existing studies mainly focusing on the use of skills checklists to help decrease medical errors and increase adherence to safety protocol. ${ }^{5-7}$ This focus on errors and safety isn't surprising given that skills checklists are effective for preventing slips in schematic behavior, that is, tasks performed reflexively or "on autopilot" 8,9 Skills checklists help by preventing errors due to concentration lapses, fatigue, or distractions. ${ }^{8}$

Skills checklists also help combat the forgetting curve. German psychologist, Hermann Ebbinghaus, first discussed the forgetting curve over a century ago $^{10}$, when he studied how humans lose their memory of knowledge over time unless the learners repeatedly review or apply the knowledge. Knowledge retention starts at $100 \%$ when one first learns the material and often slopes downward to just $40 \%$ after only a couple of days. Tools such as checklists require the learner to retrieve the knowledge after learning which helps sustain knowledge retention.1112

Tasks requiring more attentional behavior that consist of problem-solving and active planning may need solutions beyond a supervisor skills checklist. ${ }^{8}$ An alternative to the supervisor skills checklist is the independent skills checklists that also includes self-reflection, or "self-monitoring checklist," a term used in the field of education and one that most closely describes the tools discussed in this paper. ${ }^{13-15}$ While self-monitoring is not a reliable way to measure the impact of training, it can be a tool to implement the skills taught in training.$^{16}$ Areas of increasing importance in healthcare such as cultural competence and integrated care require a better understanding of one's life view as well as improving communication skills. The studies discussed here use a self-monitoring checklist as a tool for increasing awareness of one's attitudes and competencies, motivated by previous findings that an increase in awareness is an important step toward successful behavior change. ${ }^{17}$ Focusing on the self also enables self-evaluation which includes comparing one's feelings and behaviors to external standards. ${ }^{17,18}$ The studies here included online training course interventions, which provided learners with the external standards for comparing oneself.
Cultural competence in healthcare is "the ability of systems to provide care to patients with diverse values, beliefs and behaviors, including tailoring delivery to meet patients' social, cultural, and linguistic needs". ${ }^{19}$ The Society of Teachers of Family Medicine guidelines for teaching cultural competence to health professionals includes an "awareness of the influences that sociocultural factors have on patients, clinicians, and the clinical relationship" and "recognition of personal biases against people of different culture" ${ }^{20}$ Self-reflection can be an important aspect of understanding personal viewpoints, enabling improved proficiency in providing culturally competent care..$^{21-23}$ Integrated care systematically coordinates general and behavioral health by defragmenting mental health, substance abuse, and primary care services. ${ }^{24}$ This fosters a team-based approach where mental health and physical health providers work together to improve care for the patient. Proficiency in areas such as cultural competence and integrated care is largely skills-based and dependent on selfawareness and communication. This paper examines the use of self-monitoring checklists for bridging the gap between skills training and skills practice in two randomized controlled trials targeting healthcare professionals, one focused on cultural competence and another focused on integrated care.

\section{Study 1: Cultural competence}

\section{Methods}

Study participants were 55 employees from two community-based behavioral healthcare organizations and one post-acute healthcare organization in the U.S. who were invited by the individual in charge of training at their organization to join the study. The invitation included that participating in the study was voluntary and would not affect their employment. The positions held by the employees varied and included providers, other clinicians, direct support professionals, executives, and administrative personnel. The majority of participants were white, female, and between 25 to 54 years old. The Center for Outcome Analysis Human Subjects Division reviewed and approved this study.

Participants were randomly assigned to an intervention or control group using a computer randomization function. The intervention consisted 
of two main components: 1) Five online courses focused on cultural competency, and 2) Completion of three online self-monitoring checklists with no employer supervision, one for each of three clients of the participant's choosing. The courses, developed by Relias (https://www.relias.com), were completed online and required about eight and a half hours of training time. Course topics addressed cultural diversity, advocacy and multicultural care, working effectively with gender and sexual minorities, infusion of culturally responsive practices, and basic communication and conflict management skills. Self-reflection items from the online tool are shown in Figure 1. Intervention group participants were directed to complete three of these tools at any point during the study and with three clients of their choosing. Participants who were not clinicians were instructed to complete the tool considering three coworkers of their choosing. The control group received a link via email with information sourced from the Health Resources and Services Administration (HRSA) about culture, language, and health literacy. The control group had the option to receive the online training at the end of the study.
Participants completed online assessments at pre-intervention, at two months after starting the study at post-intervention, and at six weeks followup after post-intervention that each took about ten minutes to complete and measured knowledge and attitudes. Participants were told at the start of the study that they would receive a USD20.00 Amazon gift card if they completed all three assessments. There were 21 questions on each assessment measuring knowledge from the courses. These questions were developed by subject matter experts who had extensive experience writing course content and questions. The questions related to attitudes regarding cultural competence were from the valid and reliable Cultural Competence Assessment Instrument (CCAI) ${ }^{25,26}$, slightly modified to fit the scope of this study. The post-intervention assessment also included a question asking about the degree of helpfulness of the self-monitoring checklist tool. Data were analyzed with SPSS version 24 (IBM Corp. Armonk, New York) and StataSE version 15 (StataCorp LP, College Station, Texas, USA) using repeated measures ANOVA.

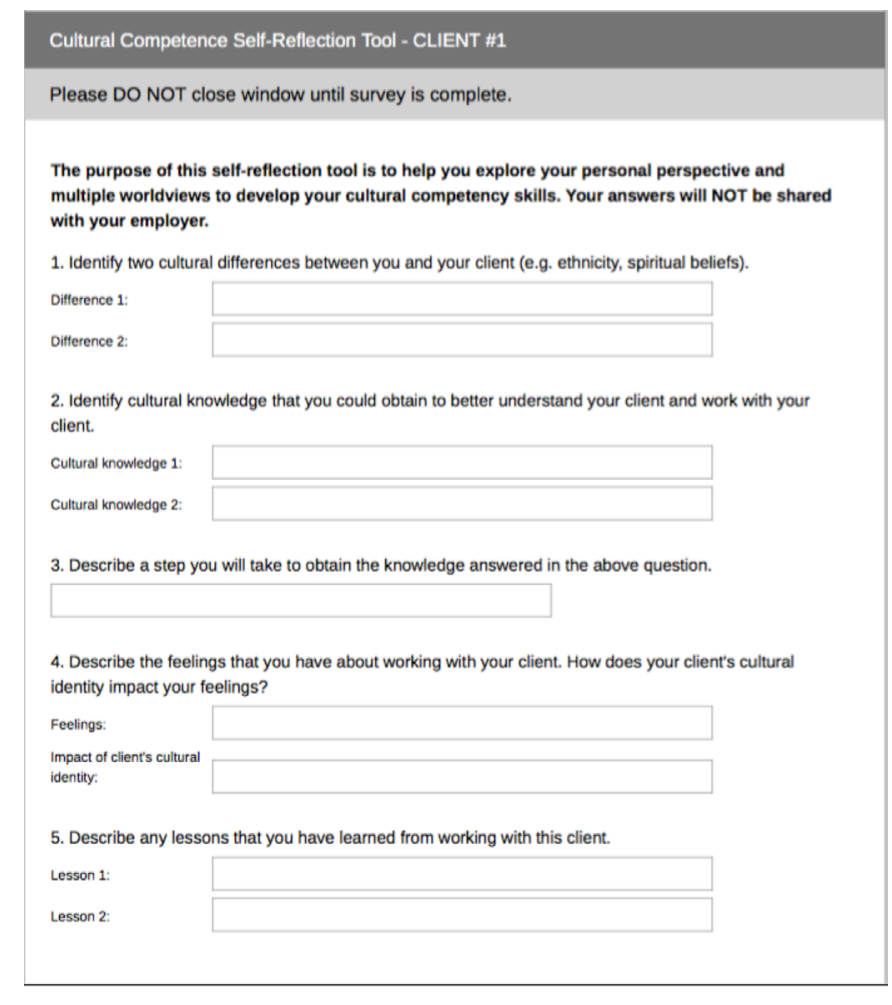

Figure 1. Cultural competence tool 


\section{Results}

The compliance rate for completing all three assessments was $53 \%$, with approximately the same compliance rate for both the intervention and control groups. For knowledge scores, there was not a main effect for time, $(p=0.331)$, group $(p=0.545)$ or training effect interaction $(p=0.934)$. There were also no significant differences in attitude scores over time. On the post-assessment, 91\% of the intervention group responders reported finding the self-monitoring checklists were helpful.

\section{Study 2: Integrated care}

\section{Methods}

Study participants were 65 behavioral health professionals recruited from a behavioral healthcare company in the Southeastern U.S. Participants were invited by their manager to join the study. The invitation included that participating in the study was voluntary and would not affect their employment. Most of the participants were non-white, female, and professional counselors by training. The average age of the participants was 44.5 years. The health professionals were members of one of six Assertive Community Treatment (ACT) teams that provide highly-individualized comprehensive treatment to people with mental illness in their own home and community. Three of the ACT teams were randomly assigned using a computer randomization function to an intervention group and the other three teams to a control group. Randomization by site was more feasible for the participating company and decreased the chances of group contamination. The Institutional Review Board at Arizona State University reviewed and approved the study protocols. The intervention consisted of two main components: 1) Four online courses focused on integrated care or diabetes targeting the healthcare professional, and 2) Completion of three online self-monitoring tools anonymously and based on three different clients of the participant's choosing. The courses, developed by Relias (https://www.relias.com), were completed online and required about six and a half hours of training time. Course topics addressed diabetes, communication with medical providers and medical terminology, nutrition and exercise for clients in behavioral health, and overweight and obesity in individuals with mental illness. The online self-monitoring checklists focused on five main diabetes standards of care-- retinal, urinalysis, A1C, lipid profiles, and foot exam. Participants completed ten items on each self-monitoring checklist which included five questions about whether they educated their client and five questions about whether they communicated with the medical provider for each of the five standards of care. Participants were instructed to complete three self-monitoring checklists at any point during the two-month intervention for any three clients of their choosing (Figure 2). The control group received an online link to information about integrated care but did not receive the courses or the online self-monitoring tool until after the study was finished.

Participants in both the intervention and control groups completed three online assessments-one at the start of the study, one after two months at the end of the intervention, and one at five-months follow-up that each took about ten minutes to complete. Participants were told at the start of the study that they would be entered in a drawing for one of three Amazon gift cards once they completed all three assessments. Each of the assessments included eight questions about content from the courses to examine knowledge, two questions about interaction with medical care providers to examine communication, and two questions about which ACT team they were from and what general job level for demographic purposes. The questions related to course content were written by subject matter experts with terminal degrees, extensive online course development experience, and multiple research publications. The questions related to interaction with medical care providers were adapted to an individual level from survey tools in integrated care where the focus has mainly been on healthcare systems as opposed to individual providers. The post-intervention assessment also included a question asking about the degree of helpfulness of the self-monitoring checklist tool. All assessments were tracked using an anonymous reproducible identifier consisting of six digits (first three digits of mother's name and first three digits of phone number).

The data were examined using two mixed effects models: a no-growth, or intercept only, model that assumes there is no change over time and a conditional growth model which assumes there is 
Please print and complete this checklist for three patients with diabetes. This is for your personal use and will not be evaluated by your supervisor or the researchers.

\begin{tabular}{|l|l|l|l|}
\hline Met & $\begin{array}{l}\text { Not } \\
\text { Met }\end{array}$ & \multicolumn{1}{|c|}{ Title } & \multicolumn{1}{c|}{ Description } \\
\hline & & Retinal & Educate client and family about receiving retinal. \\
\hline & & Retinal & $\begin{array}{l}\text { If client has not received retinal, communicate with primary } \\
\text { care physician about receipt of retinal. }\end{array}$ \\
\hline & & Urinalysis & Educate client and family about receiving urinalysis. \\
\hline & & Urinalysis & $\begin{array}{l}\text { If client has not received urinalysis, communicate with } \\
\text { primary care physician about receipt of urinalysis. }\end{array}$ \\
\hline & & 2A1C & Educate client and family about receiving 2A1C. \\
\hline & & 2A1C & $\begin{array}{l}\text { If client has not received 2A1C, communicate with primary } \\
\text { care physician about receipt of 2A1C. }\end{array}$ \\
\hline & & Lipid profiles & Educate client and family about receiving lipid profiles. \\
\hline & Lipid profiles & $\begin{array}{l}\text { If client has not received lipid profiles, communicate with } \\
\text { primary care physician about receipt of lipid profiles. }\end{array}$ \\
\hline & & Foot exam & Educate client and family about receiving foot exam. \\
\hline & & Foot exam & $\begin{array}{l}\text { If client has not received foot exam, communicate with } \\
\text { primary care physician about receipt of foot exam. }\end{array}$ \\
\hline
\end{tabular}

Figure 2. Integrated Care Tool

change over time that depends on a conditional factor, in this case, the intervention group. A curvilinear effect of time was used, which was measured in weeks, because of expectations that the effects would increase after training and remain stable over time. Analyses were performed using SPSS version 24 (IBM Corp. Armonk, New York).

\section{Results}

All 65 invited participants completed the study. The conditional growth model with a quadratic effect of time showed a significantly better fit than the no-growth model, $\mathrm{p}<0.001$, and demonstrated a significant increase in knowledge over time for the intervention group compared to the control group in a curvilinear fashion, $p<0.001$, improving steeply between baseline and post-test, and then leveling off. Compared to the control group, the intervention group showed more improvement in knowledge scores between baseline and post-test and that knowledge level remained stable at five months follow up. Neither the intervention or control group show significant changes in communication from baseline to post-intervention or from postintervention to follow-up. On the post-assessment,
$90 \%$ of the intervention group participants reported finding the self-monitoring checklists were "very helpful" or "somewhat helpful."

\section{Discussion}

The two studies presented here indicate that self-monitoring checklists may be a useful tool for helping to bridge the gap between training and practice. The vast majority of learners in both studies found self-monitoring checklists helpful, and the assessment results in Study 2 suggest self-monitoring checklists may be effective for helping learners improve and sustain knowledge over time. This is consistent with other studies that have shown skills checklists to be helpful for preventing errors and increasing adherence to safety measures. ${ }^{5,6}$ The findings herein are unique in that there were no supervisors overseeing completion of the online tool and it also included self-reflection.

There are several applications in healthcare settings for using self-monitoring checklists. The online aspect of the self-monitoring checklists used in this study makes it a tool available to a health professional with access to a computer 
or mobile device. Furthermore, the learners can complete the self-monitoring checklists at a time convenient to their work schedules rather than needing to be physically present at a certain time and place. Another potential application of selfmonitoring checklists is as memory aids since learners forget much of the content learned in training. ${ }^{11,12}$

\section{Limitations}

There are several limitations to these studies. First, there was poor compliance with the assessments in Study 1. One possible reason for worse compliance in Study 1 may be that there were two more hours of coursework in that study than in Study 2. Additionally, the executive team at the company for Study 2 participants showed strong support of the study to its employees by communicating the study's importance and posting reminders to participate. Second, the helpfulness of the selfmonitoring checklists and the change in skills was based on self-reported data. Studies show that selfreported data may contain biases. ${ }^{27}$ In this study, in an effort to prevent biases, the researchers notified participants that their employers would not see their individual answers and Study 2 went even further by using an anonymous ID. Third, due to feasibility and effort to keep intervention and control group participants from discussing the online training with each other, the participants were randomized by ACT team for Study 2 . There is a chance that a factor related to their ACT team may have influenced the outcomes. When asked about any potential factors unique to any ACT teams that may have influenced the outcome, the company had none to report.

\section{Conclusion}

With the increasing importance of patient experience and patient-centered care in the field of medicine, healthcare professionals must improve their "softer" skills in areas such as cultural competence and communication with providers and clients. Tools such as the self-monitoring checklists described in this paper can help bridge the gap between training on these topics and implementation into practice. Future studies are recommended examining actual patient outcomes after healthcare professionals have used self-monitoring checklists in conjunction with online training programs. For now, educators and training developers should keep self-monitoring checklists in mind as low-cost and low-resource intensive tools for helping healthcare professionals incorporate their training into practice.

Conflicts of interest: All authors are employees of Relias. The courses used in this study were developed by Relias for use in their commercial activities.

Funding sources: None.

\section{References}

1. Brake J. Bridging the gap between knowledge and practice. J Diabetes Nurs. 2005 Mar;9(3):9296.

2. Doehring P. Autism services across America: road maps for improving state and national education, research, and training programs. Baltimore, MA: Paul H. Brookes Publishing Co.; 2013. 333 p.

3. Morton A, Wigley W. Beyond 'train and hope': identifying factors that affect implementation of the Promotional Guide in practice. J Health Visit. 2014;2(12):670-680. doi: 10.12968/ johv.2014.2.12.670

4. Hales BM, Pronovost PJ. The checklist--a tool for error management and performance improvement. J Crit Care. 2006 Sep;21(3):231-5. doi: 10.1016/j.jcrc.2006.06.002

5. Clay-Williams R, Colligan L. Back to basics: checklists in aviation and healthcare. BMJ Qual Saf [Internet]. 2015 [cited 2018 Nov 20]; 24(7):42831. Available from: https://doi.org/10.1136/ bmjqs-2015-003957

6. Hales B, Terblanche M, Fowler R, Sibbald W. Development of medical checklists for improved quality of patient care. Int J Qual Health Care. 2008 Feb;20(1):22-30. doi: 10.1093/intqhc/mzm062

7. Bosk CL, Dixon-Woods M, Goeschel CA, Pronovost PJ. Reality check for checklists. Lancet. 2009 Aug 8;374(9688):444-5.

8. Checklists [Internet]. [place unknown]: U. S. Department of Health and Human Services; [updated 2018 Aug; cited 2018 Nov 22]. Available from: https://psnet.ahrq.gov/primers/primer/14

9. Hilligoss B, Moffatt-Bruce SD. The limits of checklists: handoff and narrative thinking. BMJ 
Qual Saf. 2014 Jul;23(7):528-33. doi: 10.1136/ bmjas-2013-002705

10. Ebbinghaus H. Memory: a contribution to experimental psychology. Ruger HA, Bussenius $\mathrm{CE}$, translators. New York, NY: Dover Publications; 1913. $123 \mathrm{p}$.

11. Karpicke JD, Grimaldi PJ. Retrieval-based learning: a perspective for enhancing meaningful learning. Educ Psychol Rev. 2012;24(3):401-418. doi: 10.1007/ s10648-012-9202-2

12. Storm BC, Bjork RA, Storm JC. Optimizing retrieval as a learning event: when and why expanding retrieval practice enhances long-term retention. Mem Cognit. 2010 Mar;38(2):244-53. doi: 10.3758/ mc.38.2.244

13. Peters EE. Developing content knowledge in students through explicit teaching of the nature of science: influences of goal setting and selfmonitoring. Sci Educ. 2012 Jun 1;21(6):881-898. doi: 10.1007/s11191-009-9219-1

14. Oliver RM, Wehby JH, Nelson JR. Helping teachers maintain classroom management practices using a self-monitoring checklist. Teaching and Teacher Education. 2015;51:113-120. doi: 10.1016/j. tate.2015.06.007

15. Ganz J. Self-monitoring across age and ability levels: teaching students to implement their own positive behavioral interventions. Prev Sch Fail. 2008;53(1):39-48.

16. Davis DA, Mazmanian PE, Fordis $M$, Van Harrison $\mathrm{R}$, Thorpe KE, Perrier L. Accuracy of physician self-assessment compared with observed measures of competence: a systematic review. JAMA. 2006 Sep 6;296(9):1094-102. doi: 10.1001/ jama.296.9.1094

17. Duval S, Wickland RA. A theory of objective self awareness. New York, NY: Academic Press; 1972. $238 \mathrm{p}$.

18. Silvia PJ, Duval TS. Objective self-awareness theory: Recent progress and enduring problems. Pers Soc Psychol Rev. 2001;5(3):230-241. doi: 10.1207/S15327957PSPR0503 4

19. Betancourt JR, Green AR, Carrillo JE. Cultural competence in health care: emerging frameworks and practical approaches. New York, NY: Commonwealth Fund, Quality of Care for Underserved Populations; 2002. 30 p.
20. Like RC, Steiner RP, Rubel AJ. STFM Core Curriculum Guidelines. Recommended core curriculum guidelines on culturally sensitive and competent health care. Fam Med. 1996 Apr;28(4):291-7.

21. Briscoe L. Becoming culturally sensitive: a painful process? Midwifery. 2013 Jun;29(6):559-65. doi: 10.1016/j.midw.2011.08.017

22. Pilcher ES, Charles LT, Lancaster CJ. Development and assessment of a cultural competency curriculum. J Dent Educ. 2008 Sep;72(9):1020-8.

23. White AA, 3rd, Logghe HJ, Goodenough DA, Barnes LL, Hallward A, Allen IM, et al. Selfawareness and cultural identity as an effort to reduce bias in medicine. J Racial Ethn Health Disparities. 2018 Feb;5(1):34-49. doi: 10.1007/ s40615-017-0340-6

24. SAMHSA-HRSA Center for Integrated Health Solutions [Internet]. [place unknown]: U. S. Department of Health and Human Services; [cited 2018 Nov 22]. Available from: https://www. integration.samhsa.gov/

25. Balcazar F, Suarez-Balcazar Y, Taylor-Ritzler T, Rodakowski J, Willis C, Portillo N. Cultural competence assessment instrument (CCAI). Chicago, IL: University of Illinois at Chicago; 2009. Available from: http://ccbmdr.ahslabs.uic.edu/wpcontent/uploads/sites/5/2014/04/CCAl.pdf

26. Suarez-Balcazar Y, Balcazar F, Taylor-Ritzier T, Portillo N, Rodakowsk J, Garcia-Ramirez M, et al. Development and validation of the cultural competence assessment instrument: a factorial analysis. J Rehabil. 2011 Jan;77(1):4-13.

27. Stone AA, Turkkan JS, Bachran C, Jobe JB, Kurtzman HS, Cain VS. The science of self-report: implications for research and practice. Mahwah, NJ: Lawrence Erlbaum; 2000. 380 p. 